\title{
Electron Diffraction Studies of Electrode Pretreatment
}

\author{
T. C. Franklin*, Marvin Beasley*, M. Petracek*, \\ T. Itoh** and J. Barrett*** \\ * Chemistry Department, Baylor University Waco, Texas. \\ ** Toa Nenryo Kogyo K.K., Central Research Laboratory, Ohi-mura, Saitama-ken, Japan. \\ *** Stamford University Birmingham, Alabama, U.S.A.
}

Received Aug. 10, 1967

\begin{abstract}
Electron diffraction studies indicated that the aging of freshly deposited nickel or activated platinum was at least in part caused by changes in crystal structure. Confirmation was also obtained for the idea that the different forms of hydrogen adsorbed on platinum are hydrogens adsorbed on different crystal planes.
\end{abstract}

It is well recognized that the results obtained with the early runs on a freshly prepared catalyst or electrode surface are quite different from those obtained in -later runs or at longer times (1-10). The two most common explanations of this aging process are the adsorption of poisons or a change in crystal structure.

The activity of platinized platinum electrodes is also associated with the presence on the platinum of catalytic sites with different bonding energies as shown by current voltage curves (11) and catalytic studies (12). These different sites have been attributed to the different crystal planes (13).

Because these phenomena have been associated with the crystal structure, electron diffraction studies were made of the processes to determine whether changes in crystal structure were involved.

\section{Experimental}

The electron diffraction studies were made with a Siemans Elmiskop I electron microscope. Flat samples were studied using a reflection cartridge. Transmission studies were made by focusing on the edge of a wire electrode.

Electron diffraction patterns were taken of the aging of a freshly plated nickel electrode. The nickel was plated from the following bath under the stated plating conditions: $\mathrm{NiSO}_{4} \cdot 6 \mathrm{H}_{2} \mathrm{O}, 240 \mathrm{~g}$. /1.; $\mathrm{NiCl}_{2} \cdot 6 \mathrm{H}_{2} \mathrm{O}$, 45g./1.; borid acid, $30 \mathrm{~g}$. /1.; pH, 2; current density, 6 ma./cm. ${ }^{2}$; temperature, $25^{\circ} \mathrm{C}$; plating time, 10 minutes. The plated samples were nickel foil with an exposed area of $3.5 \mathrm{~cm}^{2}$. After plating the samples were rinsed in water, absolute alcohol and then cut to fit in the electron microscope sample holder. 
The platinum aging study was made on a $0.35 \mathrm{~cm}^{2}$. sample of bright platinum foil activated in a pre-electrolyzed $2 N$ sulfuric acid solution. Oxygen was generated on the electrode for thirty minutes at a current density of $100 \mathrm{~mA} . / \mathrm{cm}^{2}$. Prepurified hydrogen was bubbled over the electorde until the potential of the electrode reached equilibrium. The hydrogen adsorbed on the electrode was polarographically oxidized to make certain (from the shape of the wave) that the electrode was active. Hydrogen was again bubbled over the electrode until equilibrium was reached. The electrode was then rinsed, cut, and inserted in the electron microscope. Similar experiments were run using platinum wire electrodes.

Activation of platinum was also accomplished by alternately oxidizing and reducting the electrode at oxygen and hydrogen evolution for several minutes. The time of oxidation or reduction on each cycle was approximately one minute.

A study was also made of the effect of electrode pretreatment on the electrodeposition of platinum black and the resulting effect on the adsorbed hydrogen and crystallinity of the deposit. The platinum was plated on a $2 \mathrm{~cm}$. wire from a solution $3 \%$ in chloroplatinic acid and $0.06 \%$ in lead acetate for 90 seconds at $15 \mathrm{ma}$. In another series of experiments, plates of similar thickness were made on platinum foils that would fit directly into the reflection attachment of the microscope. Comparison was made in each case between the behavior of platinum deposited on a mechanically polished substrate (polished with emery cloth) and platinum deposited on a chemically etched base (etched with aqua regia).

\section{Discussion of Results}

\subsection{Aging of Freshly Plated or Activated Electrodes}

The nickel sample was inserted into the microscope immediately after plating and the first picture was taken in less than two minutes after plating. Pictures were then taken at different times. When the elapsed time was less than two minutes either no rings at all were detected or only very broad diffuse rings were present. After two to five minutes in the microscope the diffuse rings had become sharp. After ten to twenty minutes the rings stopped changing in appearance giving a pattern identical to a nickel foil sample and in agreement with the A.S.T.M. pattern. Nickel plated samples that had stood for approximately one hour in water were also used as a blank in one run. This sample gave results identical to the nickel foil and A.S.T.M. patterns.

In both the reflection and transmission studies, the activated platinum samples gave no diffraction lines for the first few minutes in the electron microscope. Gradually with increasing time in the electron beam, diffraction lines developed and became sharp. The end results after 10 minutes were a diffraction pattern identical to that of a test platinum foil. An activated platinum sample after standing in solution in air for 15 minutes gave a strong crystalline pattern identical to that of platinum. Studies of the activity of the platinum as measured by its ability to adsorb hydrogen showed that in this 15 minute period the activity of the platinum had decreased sharply and had reached an activity that was changing only slowly with time.

From this data it would seem logical to conclude that freshly activated platinum or freshly plated nickel are in an amorphous state. Upon aging these metals 
crystallize. Although it seems certain that activating platinum serves to clean the electrode (10) it also appears from this data and from logic that the severe conditions of making and reducing the oxide with the concurrent changes in volume breaks the crystal bonding forces.

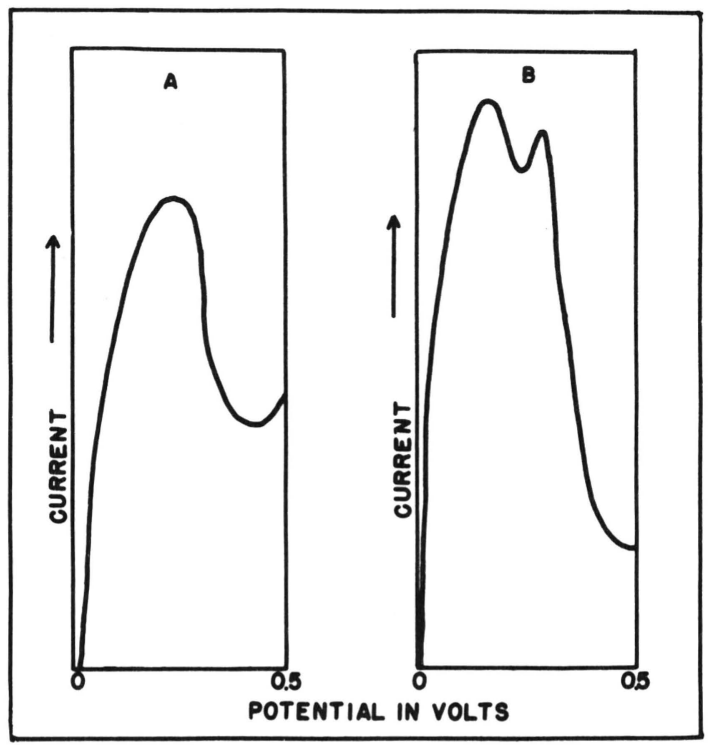

A - Platinum plated on a mechanically polished surface.

B - Platinum plated on a chemically etched surface.

Fig. 1 Typical current voltage curves for the oxidation of adsorbed hydrogen on platinized platinum in two normal sulfuric acid

\subsection{Effect of Electrode Pretreatment on the Plating of Platinum Black}

Fig. 1 shows two current voltage curves for the oxidation of hydrogen on platinized platinum in $2 N$ sulfuric acid. The presence of two maxima, indicating forms of hydrogen with different bonding energies (11), is evident in the data taken using platinum plated on an electrode that had been etched in aqua regia. On the other hand, the curve obtained when the platinum was plated on a mechanically polished substrate had only one maximum indicating the presence of only one major form of adsorbed hydrogen.

Both transmission and reflection elecron diffraction patterns of the deposits showed no diffraction lines for the deposit on the mechanically polished substrates while sharp diffraction lines were obtained for the deposits on the chemically etched substrate. These diffraction lines agreed with those obtained on platinum foil.

From these data it is logical to conclude that platinum black deposited on mechanically polished substrates is amorphous. Such a deposit would be expected to show the presence of only one type of adsorption site and this is what was observed experimentally.

On the other hand, the platinum black deposited on crystalline chemically etched platinum is crystalline and gives maxima in the current-voltage curves indicating the presence of different forms of adsorbed hydrogen. This is in agreement with Will's (13) conclusion that the different forms of hydrogen are associated with different crystal planes.

\section{Acknowledgement}

This work has been supported by The Robert A. Welch Foundation of Houston, Texas.

\section{Literature:}

1) F. C. Anson, P. M. King, Anal. Chem. 34, 362 (1962).

2) J. O'M. Bockris, I. A. Ammer, A.K.M.S. Huq, J. Phys. Chem. 61, 879 (1957).

3) J. O'M. Bockris, I. A. Azzam, Trans. Faraday Soc. 48, 154 (1952).

4) M. Breiter, B. Kennel, Z. Elektrochem. 64, 1180 (1960).

5) W. G. French, T. Kuwana, J. Phys. Chem. 68, 1279 (1964). 
6) S. Shibata, Bull. Chem. Soc. Japan 36, 525 (1963).

7) S. Shibata, J. Chem. Soc. Japan, Pure Chem. Sec. 79, 239 (1958).

8) J. L. Weininger, M. W. Breiter, J. Electrochem. Soc. 111, 707 (1964).

9) F. G. Will, C. A. Knorr, Z. Elektrochem. 64, 258 (1960).

10) S. D. James, "The Electrochemical Activation of Platinum Electrodes" Extended Abstracts of the Spring, 1967, Meeting of
The Electrochemical Society, Abstract No. 175.

11) A. N. Frumkin, Advances in Electrochemistry and Electrochemical Engineering, Vol. 3, edited by Paul Delahay, p. 307. Interscience Publishers, New York, N. Y.(1963).

12) T. C. Franklin, D. H. McClelland, J. Phys. Chem. 67, 2436 (1963).

13) F. G. Will, J. Electrochem. Soc. 112, 451 (1965).

\title{
Kinetic Study on the Sulfidation of Copper by Hydrogen Sulfide
}

\author{
Masayuki Takeda, Kazuo Fueki and Takashi Mukaibo \\ Department of Industrial Chemistry, The Faculty of Engineering, \\ The University of Tokyo, Bunkyo-ku, Tokyo
}

Received Aug. 21, 1967

The sulfidation of copper by hydrogen sulfide was studied kinetically in a temperature range of 400 to $500^{\circ} \mathrm{C}$.

It was found that the sulfidation obeyed the so-called linear law and that the linear rate constant was proportional to the partial pressure of hydrogen sulfide. It was also found that the sulfidation rate was independent of the partial pressure of hydrogen. The activation energy was $21.3 \mathrm{kcal} / \mathrm{mol}$.

Microscopic observation on the cross section and the surface of sulfide film revealed that the sulfide film is singly layered and compact.

$\mathrm{X}$-ray diffraction study showed the sulfide to be cuprous sulfide.

From these results, it is concluded that the sulfidation is controlled by the reaction at the sulfide-gas interface.

On the basis of the theory and experiment by Kobayashi and Wagner, the independence of sulfidation rate on partial pressure of hydrogen is also discussed. 